\title{
Distributed Kalman-Consensus Filtering for Sparse Signal Estimation
}

\author{
Yisha Liu, ${ }^{1}$ Haiyang Yu, ${ }^{2}$ and Jian Wang ${ }^{3}$ \\ ${ }^{1}$ School of Information Science and Technology, Dalian Maritime University, Dalian 116026, China \\ ${ }^{2}$ Research Center of Information and Control, Dalian University of Technology, Dalian 116024, China \\ ${ }^{3}$ China Nuclear Power Technology Research Institute Beijing Division, Beijing 100872, China \\ Correspondence should be addressed to Haiyang Yu; yuhaiyang08@gmail.com
}

Received 24 December 2013; Accepted 21 February 2014; Published 23 March 2014

Academic Editor: Ge Guo

Copyright (C) 2014 Yisha Liu et al. This is an open access article distributed under the Creative Commons Attribution License, which permits unrestricted use, distribution, and reproduction in any medium, provided the original work is properly cited.

\begin{abstract}
A Kalman filtering-based distributed algorithm is proposed to deal with the sparse signal estimation problem. The pseudomeasurement-embedded Kalman filter is rebuilt in the information form, and an improved parameter selection approach is discussed. By introducing the pseudomeasurement technology into Kalman-consensus filter, a distributed estimation algorithm is developed to fuse the measurements from different nodes in the network, such that all filters can reach a consensus on the estimate of sparse signals. Some numerical examples are provided to demonstrate the effectiveness of the proposed approach.
\end{abstract}

\section{Introduction}

In the past few years, the problems of sparse signal recovery have received a great deal of attention since the emergence of the new signal sampling theory which is known as compressed sampling or compressed sensing [1-3]. Using fewer observations than what is required in the Shannon sample principle, sparse signals can be recovered with overwhelming probability through solving a 1-norm minimization problem. A variety of approaches have been developed for this convex optimization problem, for example, Least Absolute Shrinkage and Selection Operator (LASSO), Basis Pursuit Denoising (BPDN), and Dantzig Selector (DS).

Recently, many researchers have attempted to deal with the sparse signal recovery problem in the classic framework of signal estimation, such as the Kalman filter. In fact, the traditional Kalman filter is not effective for the sparse signals estimation [4]; therefore some improved approaches were proposed. In [5], the problem of reconstructing time sequences of spatially sparse signals was considered. Compressed sensing was used to estimate the support set of the initial signal's transform vector, and a reduced-order Kalman filter was designed to recover the signal. Based on a pseudomeasurement technology of the underlying constrained optimization problem, Carmi et al. proposed a new Kalman filtering approach for the recovery of possibly time-varying sparse signals in [6]. In [7], an algorithm based on a hierarchical probabilistic model was proposed, which used reweighted $\ell_{1}$ minimization as its core computation and propagated second order statistics through time similar to the classic Kalman filtering.

Encouraged by the exciting progress on sensor networks and multiagent systems, the distributed technology is having an important role in many fields, such as communication, multirobot systems, and production scheduling. Recently, the study of the distributed estimation or filtering has attracted considerable research interests, and some results have been reported in the literature. In $[8,9]$, a diffusion strategy was established for the design of distributed Kalman filtering and smoothing, in which nodes communicate with their direct neighbors only, and the information is diffused across the network through a sequence of Kalman iterations and data aggregation. A class of diffusion LMS strategies was proposed for distributed estimation over adaptive networks in [10]. These methods can make the network with the ability to learn the sparse structure from the incoming data in real time and also track variations in the sparsity of the model. Recently, the consensus protocol has been introduced into distributed 
filtering problems. In [11], three types of distributed Kalman filtering algorithms were proposed. A distributed high-pass filter was used to fuse the local sensor measurements based on average consensus, such that the nodes in sensor network track the average measurement of the overall network. The algorithms were established based on the information form of Kalman filter and the analysis of stability and performance of the Kalman-consensus filter was provided in [12].

In this paper, we aim to develop a distributed filtering method to estimate a sparse signal using some measurements from a sensor network. The proposed method is established by means of the Kalman-consensus filter and the pseudomeasurement technology. The remainder of this paper is organized as follows. Section 2 gives a brief overview of the basic problems in compressed sampling and summarizes the Kalman filter with embedded pseudomeasurement equation. A dynamic parameter selection strategy is discussed as well to improve the performance. In Section 3, the Kalman filtering with embedded pseudomeasurement is rebuilt in the information form. The pseudomeasurement equation is introduced into the Kalman-consensus filter to develop a distributed sparse signal estimation algorithm. Simulation results are given in Section 4 to demonstrate the effectiveness of the proposed method. Some concluding remarks are provided in Section 5.

Notation. The notation used here is fairly standard except where otherwise stated. $\mathbf{R}$ denotes the set of real numbers, and $\mathbf{R}^{n}$ denotes the $n$ dimensional Euclidean space whose elements are $n \times 1$ column vectors. The support set of $x \in \mathbf{R}^{n}$ is defined as $\operatorname{Supp}\{x\}:=\{i \mid x(i) \neq 0\}$, and $\|x\|_{0}$ is the cardinality of $\operatorname{Supp}\{x\}$. The 1-norm and 2-norm of $x$ are defined as $\|x\|_{1}=\sum_{i=1}^{n}|x(i)|$ and $\|x\|_{2}=\sqrt{x^{T} x}$, respectively. $\operatorname{sign}(\cdot)$ is the sign function. $\mathbf{E}[\xi]$ stands for the mathematical expectation of random variable $\xi$. $\mathbf{N}\left(\mu, \sigma^{2}\right)$ stands for normal distribution with mean $\mu$ and variance $\sigma^{2}$. $\mathbf{U}_{\text {int }}[a, b]$ represents the integer uniform distribution in the interval $[a, b]$. $\operatorname{Tr} A$ denotes the trace of matrix $A$. $\|A\|_{F}=\sqrt{\operatorname{Tr} A A^{T}}$ is the Frobenius norm of matrix $A$. $\operatorname{vec}\left\{x_{1}, \ldots, x_{n}\right\}:=\left[\begin{array}{lll}x_{1}^{T} & \ldots & x_{n}^{T}\end{array}\right]^{T} \cdot \operatorname{diag}\left\{A_{1}, \ldots, A_{n}\right\}$ denotes a block-diagonal matrix whose diagonal blocks are given by $A_{1}, \ldots, A_{n} . S \geq 0$ means a real symmetric matrix $S$ is semidefinite.

\section{Sparse Signal Estimation Using Kalman Filter}

This section briefly overviews some basic concepts in compressed sensing and the method proposed in [6]. In order to recover a sparse signal, a pseudomeasurement equation is embedded into the classic Kalman filter. Some more details can be found in $[1,6,13]$.

2.1. Sparse Signals Recovery. Consider a signal $x \in \mathbf{R}^{n} \cdot x$ is sparse if $\|x\|_{0}$ is much less than $n$. Moreover, $x$ is called $s$-sparse if $\|x\|_{0}=s$. Assume that $\left\{x_{k}\right\}_{k=0}^{\infty}$ is an unknown sparse discrete-time process in $\mathbf{R}^{n} ; x_{k}$ evolves according to the following dynamic model:

$$
x_{k+1}=A_{k} x_{k}+w_{k}
$$

where $A_{k} \in \mathbf{R}^{n \times n}$ is the state transition matrix; $\left\{w_{k}\right\}_{k=0}^{\infty}$ is a zero-mean white Gaussian sequence with covariance $Q_{k} \geq 0$ and $x_{0} \sim \mathbf{N}\left(\mu_{0}, P_{0}\right)$. The $m$-dimensional linear measurement of $x_{k}$ is

$$
y_{k}=H_{k} x_{k}+v_{k}
$$

where $H_{k} \in \mathbf{R}^{m \times n}$ is the measurement matrix and $\left\{v_{k}\right\}_{k=0}^{\infty}$ is a zero-mean white Gaussian sequence with covariance $R_{k} \geq 0$. The scenario we are interested in is the underdetermined case, that is, when $m<n$.

As shown in $[1,13], x_{k}$ can be accurately recovered by solving the following optimization problem:

$$
\min _{\widehat{x}_{k} \in \mathbf{R}^{n}}\left\|\widehat{x}_{k}\right\|_{0}, \quad \text { s.t. }\left\|y_{k}-H_{k} \widehat{x}_{k}\right\|_{2}^{2} \leq \varepsilon .
$$

But the optimization problem (3) is NP-hard and cannot be solved effectively. Fortunately, it has been proved in [1] that if the measurement matrix $H_{k}$ obeys the so-called Restricted Isometry Property (RIP), the solution of (3) can be obtained with overwhelming probability by solving the following convex optimization problem:

$$
\min _{\widehat{x}_{k} \in \mathbf{R}^{n}}\left\|\widehat{x}_{k}\right\|_{1}, \quad \text { s.t. }\left\|y_{k}-H_{k} \widehat{x}_{k}\right\|_{2}^{2} \leq \varepsilon .
$$

For $s$-sparse signal $x \in \mathbf{R}^{n}$, only on the order of $m=s \log n$ samples are needed to reconstruct it.

2.2. Sparse Signal Estimation Using Kalman Filter with Pseudomeasurement. For the system given in (1) and (2), the classic Kalman filter can provide an estimate of $x_{k}$ which is the solution of the following unconstrained $\ell_{2}$ minimization problem:

$$
\min _{\widehat{x}_{k} \in \mathbf{R}^{n}} \mathbf{E}_{x_{k} \mid y_{1}, \ldots, y_{k}}\left[\left\|x_{k}-\widehat{x}_{k}\right\|_{2}^{2}\right]
$$

In [6], Carmi et al. have discussed the stochastic case of (4):

$$
\min _{\widehat{x}_{k} \in \mathbf{R}^{n}}\left\|\widehat{x}_{k}\right\|_{1} \quad \text { s.t. } \mathbf{E}_{x_{k} \mid y_{1}, \ldots, y_{k}}\left[\left\|x_{k}-\widehat{x}_{k}\right\|_{2}^{2}\right] \leq \varepsilon
$$

and its dual problem

$$
\min _{\widehat{x}_{k} \in \mathbf{R}^{n}} \mathbf{E}_{x_{k} \mid y_{1}, \ldots, y_{k}}\left[\left\|x_{k}-\widehat{x}_{k}\right\|_{2}^{2}\right] \quad \text { s.t. }\left\|\widehat{x}_{k}\right\|_{1} \leq \varepsilon^{\prime} .
$$

By constructing a pseudomeasurement equation

$$
0=\widetilde{H}_{k} x_{k}-\varepsilon^{\prime},
$$

where $\widetilde{H}_{k}=\operatorname{sign}\left(x_{k}^{T}\right) ; \varepsilon^{\prime}$ is the fictitious measurement noise assumed to obey $\mathbf{N}\left(0, \sigma^{2}\right)$, and the constrained optimization problem (7) can be solved in the framework of Kalman filter. The specific method has been summarized as CSKF-1 algorithm in [6]. 
In pseudomeasurement equation (8), the measurement matrix $\widetilde{H}_{k}$ is state dependent and it can be approximated by $\widehat{H}_{k}=\operatorname{sign}\left(\widehat{x}_{k \mid k-1}^{T}\right)$. The divergence of this approximation has been discussed by Theorem 1 in [14]. Furthermore, $\sigma$ is a tunable parameter which determines the tightness of the constraint on 1-norm of the state estimate $\widehat{x}$. In [14], the authors have proved that the performance of CSKF-1 algorithm can be improved by increasing parameter $\sigma$ and the pseudomeasurement update time $r$. However, a big $\sigma$ means a loose constraint on sparseness and thus leads to slow convergence. On the contrary, a tiny $\sigma$ can result in fast convergence, but there is a big overshoot at the beginning and an enormous steady-state error. Therefore, in this paper we employ a dynamic parameter $\sigma$ which increases over time from a specific initial value, for example, set $\sigma^{2}=a+b k$, where $a$ determines the initial convergence rate and $b$ determines the increase rate of parameter $\sigma$. By selecting the parameters $a$ and $b$ properly, the convergence rate and the steady-state error of CSKF-1 algorithm can be significantly improved. The effectiveness will be demonstrated by an example in Section 4 .

\section{Distributed Sparse Signal Estimation}

Kalman-consensus filter (KCF) is a distributed estimation algorithm in sensor networks proposed in [11]. A detailed analysis on its stability and performance is given in [12]. The KCF is developed based on the information form of Kalman filter by attaching a consensus update structure on the measurement update procedure. In this section, CSKF1 algorithm will be rebuilt in the information form of Kalman filter, and the pseudomeasurement technology will be embedded in the KCF to develop a distributed sparse signal estimation algorithm in sensor networks.

Consider a sensor network whose topology is represented by an undirected graph $\mathscr{G}=(\mathscr{V}, \mathscr{E}, \mathscr{A})$ of order $N$ with the set of nodes $\mathscr{V}=\{1,2, \ldots, N\}$, the set of edges $\mathscr{E} \subseteq \mathscr{V} \times \mathscr{V}$, and the adjacency matrix $\mathscr{A}=\left[a_{i j}\right]$ with nonnegative adjacency element $a_{i j}$. An edge of $\mathscr{G}$ is denoted by unordered pair $(i, j)$. The adjacency elements associated with the edges of the graph are positive; that is, $a_{i j}>0 \Leftrightarrow(i, j) \in \mathscr{E}$. The node $j$ is called a neighbor of node $i$ if $(i, j) \in \mathscr{E}$ and $j \neq i$. The set of neighbors of node $i$ is denoted by $\mathcal{N}_{i}$. Assume that $\mathscr{G}$ is strongly connected.

Assume that the measurement of sensor node $i$ is in accordance with the linear model (2). Denote $\bar{y}_{i, k}=\left[\begin{array}{c}y_{i, k} \\ 0\end{array}\right]$, $\bar{H}_{i, k}=\left[\begin{array}{c}H_{i, k} \\ \bar{H}_{k}\end{array}\right]$, and $\bar{v}_{i, k}=\left[\begin{array}{c}v_{i, k} \\ \varepsilon_{i}^{\prime}\end{array}\right]$. We can get the following augmented measurement equation of sensor $i$ :

$$
\bar{y}_{i, k}=\bar{H}_{i, k} x_{k}+\bar{v}_{i, k}
$$

where $H_{i, k}$ is the measurement matrix of sensor $i, v_{i, k}$ is zero-mean Gaussian white noise with covariance $R_{i, k}$, $\widetilde{H}_{k}=\operatorname{sign}\left(x_{k}^{T}\right)$, and $\varepsilon_{i}^{\prime}$ is the fictitious measurement noise which obeys $\mathbf{N}\left(0, \sigma_{i}^{2}\right)$. Defining the global measurement
$\mathscr{Y}_{k}=\operatorname{vec}\left\{\bar{y}_{1, k}, \ldots, \bar{y}_{N, k}\right\}, \mathscr{V}_{k}=\operatorname{vec}\left\{\bar{v}_{1, k}, \ldots, \bar{v}_{N, k}\right\}, \mathscr{H}_{k}=$ $\operatorname{vec}\left\{\bar{H}_{1, k}, \ldots, \bar{H}_{N, k}\right\}$, and $\mathscr{R}_{k}=\operatorname{diag}\left\{R_{1, k}, \ldots, R_{N, k}\right\}$, we have

$$
\mathscr{Y}_{k}=\mathscr{H}_{k} x_{k}+\mathscr{V}_{k} \text {. }
$$

The state estimates of (1) from Kalman filter based on $\left\{\mathscr{Y}_{1}, \ldots, \mathscr{Y}_{k}\right\}$ can be defined as

$$
\begin{aligned}
\widehat{x}_{k \mid k-1} & =\mathbf{E}\left\{x_{k} \mid \mathscr{Y}_{1}, \ldots, \mathscr{Y}_{k-1}\right\}, \\
\widehat{x}_{k \mid k} & =\mathbf{E}\left\{x_{k} \mid \mathscr{Y}_{1}, \ldots, \mathscr{Y}_{k}\right\},
\end{aligned}
$$

and the estimate error covariance matrices can be obtained by

$$
\begin{gathered}
P_{k \mid k-1}=\mathbf{E}\left\{\left(x_{k}-\widehat{x}_{k \mid k-1}\right)\left(x_{k}-\widehat{x}_{k \mid k-1}\right)^{T} \mid \mathscr{Y}_{1}, \ldots, \mathscr{Y}_{k-1}\right\}, \\
P_{k \mid k}=\mathbf{E}\left\{\left(x_{k}-\widehat{x}_{k \mid k}\right)\left(x_{k}-\widehat{x}_{k \mid k}\right)^{T} \mid \mathscr{Y}_{1}, \ldots, \mathscr{Y}_{k}\right\} .
\end{gathered}
$$

The inverses of estimate error covariance matrices, $\Sigma_{k \mid k-1}=$ $P_{k \mid k-1}^{-1}$ and $\Sigma_{k \mid k}=P_{k \mid k}^{-1}$, are known as information matrices. According to the information form of Kalman filter, defining

$$
\begin{gathered}
z_{k}=\mathscr{H}_{k}^{T} \mathscr{R}_{k}^{-1} \mathscr{Y}_{k}=\sum_{i=1}^{N} H_{i, k}^{T} R_{i, k}^{-1} y_{i, k}, \\
S_{k}=\mathscr{H}_{k}^{T} \mathscr{R}_{k}^{-1} \mathscr{H}_{k}=\sum_{i=1}^{N} H_{i, k}^{T} R_{i, k}^{-1} H_{i, k}+\sum_{i=1}^{N} \sigma_{i}^{-1} \Omega_{k},
\end{gathered}
$$

where $\Omega_{k}=\operatorname{sign}\left(x_{k}\right) \operatorname{sign}\left(x_{k}^{T}\right)$, we can get

$$
\Sigma_{k \mid k}=\Sigma_{k \mid k-1}+S_{k} .
$$

The state estimate from the filter is

$$
\widehat{x}_{k \mid k}=\widehat{x}_{k \mid k-1}+\Sigma_{k \mid k}^{-1}\left(z_{k}-S_{k} \widehat{x}_{k \mid k-1}\right),
$$

and the time update procedure is

$$
\begin{gathered}
\widehat{x}_{k+1 \mid k}=A_{k} \widehat{x}_{k \mid k}, \\
\Sigma_{k+1 \mid k}^{-1}=A_{k} \Sigma_{k \mid k}^{-1} A_{k}^{T}+Q_{k} .
\end{gathered}
$$

Equations (13)-(18) have defined the Kalman information filter with embedded pseudomeasurement.

Remark 1. From (14) and (15), it is obvious that the introduction of pseudomeasurement equation impacts on the evolution of state error covariance by the sparseness of state since $\operatorname{Tr} \Omega_{k}=\left\|x_{k}\right\|_{0}$. As mentioned in Section 2, $\Omega_{k}$ will be approximated by $\widehat{\Omega}_{k}=\operatorname{sign}\left(\widehat{x}_{k \mid k-1}\right) \operatorname{sign}\left(\widehat{x}_{k \mid k-1}^{T}\right)$ in applications.

Based on the conclusion of Theorem 2 in [12], we can construct the following Kalman-consensus information filter 

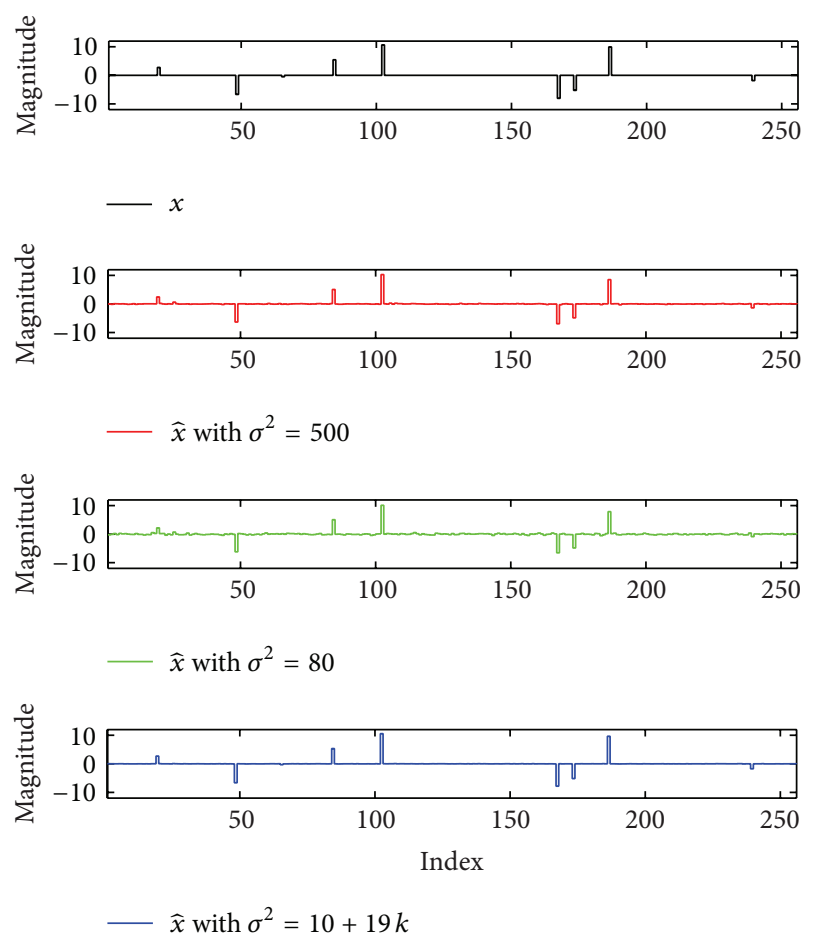

FIGURE 1: Sparse signal $x$ and its estimate $\widehat{x}$ using CSKF-1 algorithm with different $\sigma^{2}$.

with embedded pseudomeasurement (KCIF-PM) on each node in the network:

$$
\begin{gathered}
z_{i, k}=\sum_{j \in \mathcal{N}_{i}} H_{j, k}^{T} R_{j, k}^{-1} y_{j, k}, \\
S_{i, k}=\sum_{j \in \mathcal{N}_{i}} H_{j, k}^{T} R_{j, k}^{-1} H_{j, k}+\sum_{j \in \mathcal{N}_{i}} \sigma_{j}^{-1} \Omega_{k}, \\
\sum_{i, k \mid k}=\sum_{i, k \mid k-1}+S_{i, k}, \\
\widehat{x}_{i, k \mid k}=\widehat{x}_{i, k \mid k-1}+\sum_{i, k \mid k}^{-1}\left(z_{i, k}-S_{i, k} \widehat{x}_{i, k \mid k-1}\right) \\
+\gamma_{i} \sum_{i, k \mid k-1}^{-1} \sum_{j \in \mathcal{N}_{i}}\left(\widehat{x}_{j, k \mid k-1}-\widehat{x}_{i, k \mid k-1}\right), \\
\hat{x}_{i, k+1 \mid k}=A_{k} \widehat{x}_{i, k \mid k}, \\
\sum_{i, k+1 \mid k}^{-1}=A_{k} \sum_{i, k \mid k}^{-1} A_{k}^{T}+Q_{k},
\end{gathered}
$$

where $\gamma_{i}=\epsilon /\left(1+\left\|\sum_{i, k \mid k-1}^{-1}\right\|_{F}\right)$ and $\epsilon$ is a tunable parameter which determines the weight of the consensus update, such that the error dynamics is globally asymptotically stable and all filters asymptotically reach a consensus on state estimates; that is, $\widehat{x}_{1}=\widehat{x}_{2}=\cdots=\widehat{x}_{N}$.

Remark 2. The global sensing matrix $\mathscr{H}_{k}$ is assumed to satisfy the Restricted Isometry Property and the $m$-dimensional global measurement $\mathscr{Y}_{k}$ is obtained through the sensor network. It is important that, for any one node in the network, there is no constraint on the dimension of its measurement, which means it can be less than the lower bound $s \log n$.
Even though each node can only get a one-dimensional measurement, we can still recover the signal as long as the global measurement meets the lower bound and there are enough communications between nodes. This will be verified by an example in the next section.

Remark 3. Unlike CSKF-1 algorithm, there are no iterative pseudomeasurement updates in the filter. This procedure is implemented simultaneously with the consensus update during the communications between the nodes in the network.

The intact version of the algorithm given by (19)-(24) is summarized as Algorithm 1.

\section{Illustrative Examples}

In this section, we will verify the effectiveness of the methods proposed in the previous sections by some numerical examples.

4.1. Sparse Signal Estimation Using CSKF-1 with Dynamic Parameter $\sigma^{2}$. In this part, we will test CSKF-1 algorithm with two constant parameters $\sigma^{2}$ and a dynamic parameter $\sigma^{2}$ which varies according to a linear function of time. Here, we attempt to estimate a static sparse signal $x \in \mathbf{R}^{256}$ which is 10 sparse. For system (1), we have $A=I_{256}$ and $Q_{k}=0$. The index $i$ in $\operatorname{Supp}\{x\}$ and the values of $x(i)$ are unknown. The sparse signal $x$ is generated by creating the support set from $i \sim \mathbf{U}_{\text {int }}[1,256]$ and setting the value $x(i) \sim \mathbf{N}(0,5)$. The sensing matrix $H \in \mathbf{R}^{72 \times 256}$ consisted of entries sampled according to $\mathbf{N}(0,1 / 72)$. This type of matrix has been shown to satisfy the Restricted Isometry Property with overwhelming probability. The measurement noise is $v \sim \mathbf{N}\left(0,0.01^{2} \times I_{72}\right)$. Set $\widehat{x}_{0}=0$; define the a posteriori estimation error $e_{k}=x-\widehat{x}_{k \mid k}$. The parameter $\sigma^{2}$ is set to be 500,80 , and $10+19 k$, respectively. The results are shown in Figures 1 and 2 . The actual signal $x$ and its estimates with parameter $\sigma^{2}=500,80$, and $10+19 k$ are shown in Figure 1 , respectively. It is evident that the best estimate of $x$ can be obtained with the dynamic parameter $\sigma^{2}=10+19 k$. The evolutions of $\|\hat{x}\|_{1}$ and $\|e\|_{2}$ are shown in Figure 2 . These results imply that the dynamic parameter $\sigma$ with properly selected $a$ and $b$ can lead to superior performance of CSKF-1 algorithm, on both the rate of convergence and the steadystate error.

4.2. Sparse Signal Estimation Using KCIF-PM in Grid Topology Network. In this part, we will estimate a sparse signal using Algorithm 1, and all of measurements are obtained through a sensor network. Without loss of generality, consider a sensor network with 6 nodes as shown in Figure 3, whose topology is represented by an undirected graph $\mathscr{G}=(\mathscr{V}, \mathscr{E}, \mathscr{A})$ with the set of nodes $\mathscr{V}=\{1,2,3,4,5,6\}$, the set of edges $\mathscr{E}=$ $\{(1,2),(1,3),(2,3),(2,4),(2,5),(3,5),(3,6),(4,5),(5,6)\}$, and the adjacency matrix: 


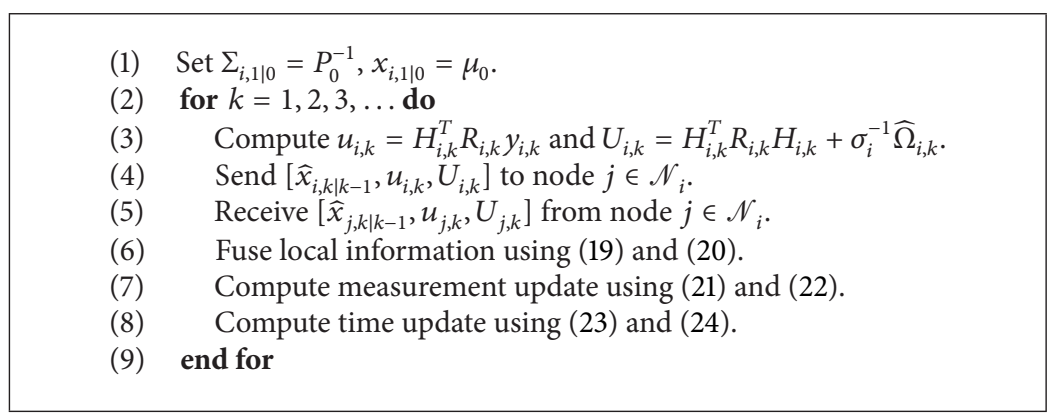

Algorithm 1: KCIF-PM.
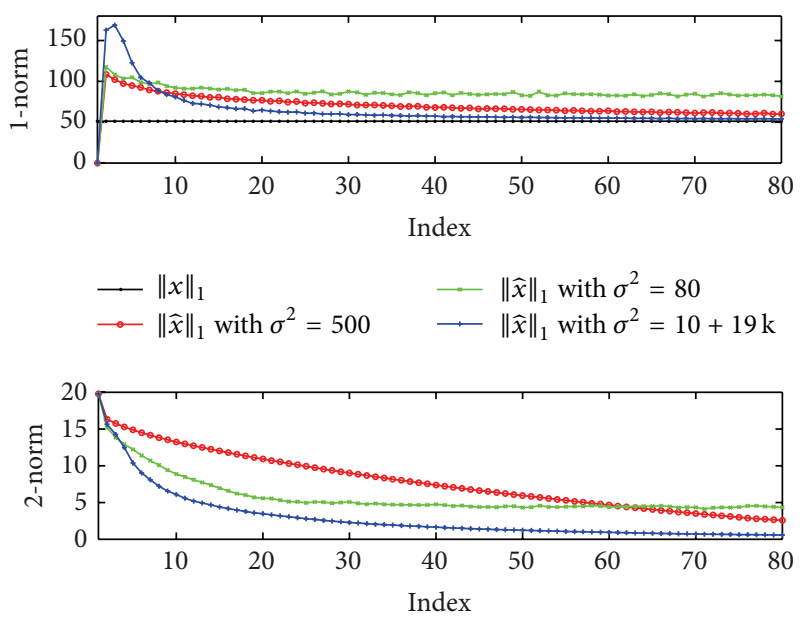

$\rightarrow\|e\|_{2}$ with $\sigma^{2}=500 \quad-\|e\|_{2}$ with $\sigma^{2}=10+19 k$
$\because\|e\|_{2}$ with $\sigma^{2}=80$

Figure 2: The evolutions of $\|\widehat{x}\|_{1}$ and $\|e\|_{2}$ with different $\sigma^{2}$.

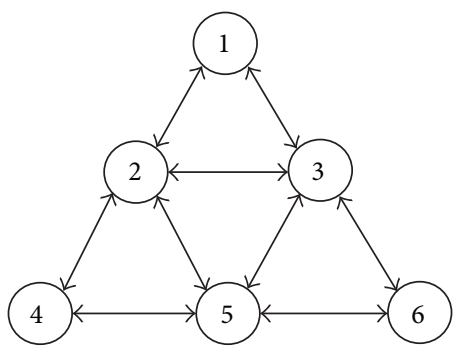

Figure 3: Topology structure of the sensor network.

$$
\mathscr{A}=\left[\begin{array}{llllll}
1 & 1 & 1 & 0 & 0 & 0 \\
1 & 1 & 1 & 1 & 1 & 0 \\
1 & 1 & 1 & 0 & 1 & 1 \\
0 & 1 & 0 & 1 & 1 & 0 \\
0 & 1 & 1 & 1 & 1 & 1 \\
0 & 0 & 1 & 0 & 1 & 1
\end{array}\right]
$$

In this simulation, the 72-dimensional measurements are obtained through the network. Here we assume that each node can get a 12-dimensional measurement, that is, $H_{i} \in$ $\mathbf{R}^{12 \times 256}$ with its entries sampled according to $\mathbf{N}(0,1 / 72)$. Set $\widehat{x}_{i, 0 \mid 0}=0, \sigma_{i}^{2}=5+17 k$, and $\epsilon=1$. The performance
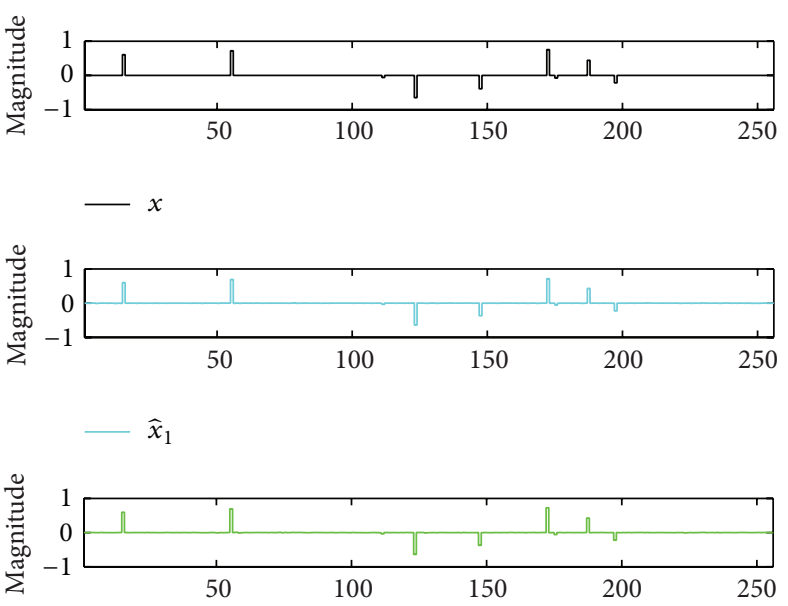

$-\widehat{x}_{2}$

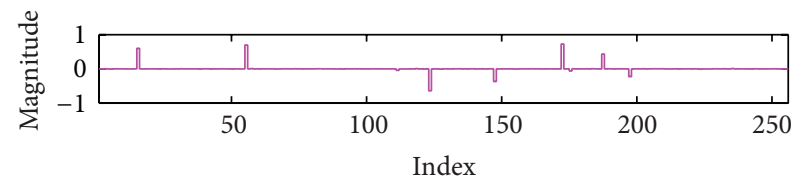

$-\widehat{x}_{3}$

FIGURE 4: The sparse signal $x$ and its estimates $\widehat{x}_{i}(i=1,2,3)$ using Algorithm 1.

of Algorithm 1 is depicted by Figures $4-6$. Figures 4 and 5 present the actual signal $x$ and its estimates $\widehat{x}_{i}(i=1,2, \ldots, 6)$ from all filters. The 1-norm of $x$ and $\widehat{x}_{i}(i=1,2, \ldots, 6)$ are shown in the top figure of Figure 6 . The bottom figure of Figure 6 presents the 2 -norm of errors $e_{i}(i=1,2, \ldots, 6)$. These results show that the Kalman-consensus filter with pseudomeasurement can fuse the measurements from the nodes, and satisfactory estimates can be obtained for the actual sparse signal recovery. It should be noted that, for any single node, the measurement dimension is not necessary to satisfy the lower bound $s \log n$. For instance, in the above example, the lower bound on the dimension of measurement is $10 \log 256 \approx 56$, but only 12 measurements are obtained by each node. The estimation accuracy of Algorithm 1 is determined by the global measurement obtained from the whole network, and the convergence speed is influenced by the topology structure of the network. 

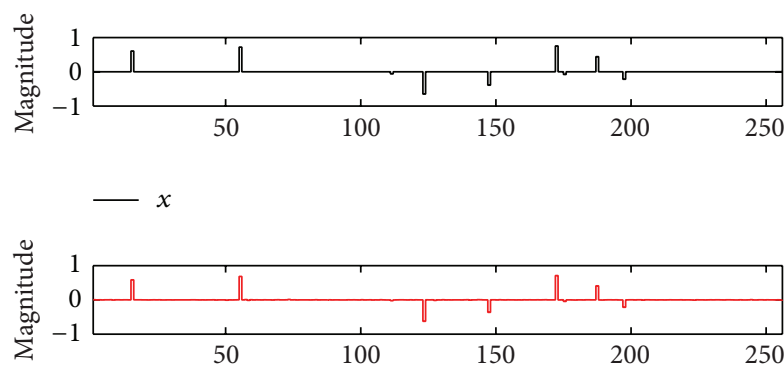

$-\widehat{x}_{4}$

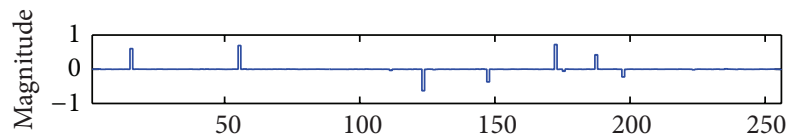

$-\widehat{x}_{5}$

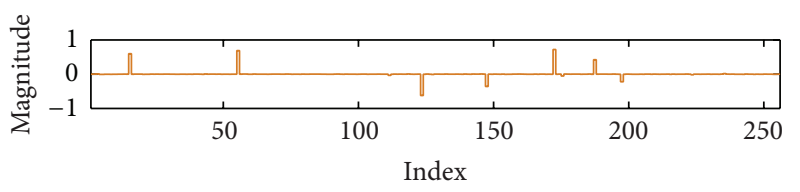

$-\widehat{x}_{6}$

FIGURE 5: The sparse signal $x$ and its estimates $\widehat{x}_{i}(i=4,5,6)$ using Algorithm 1.
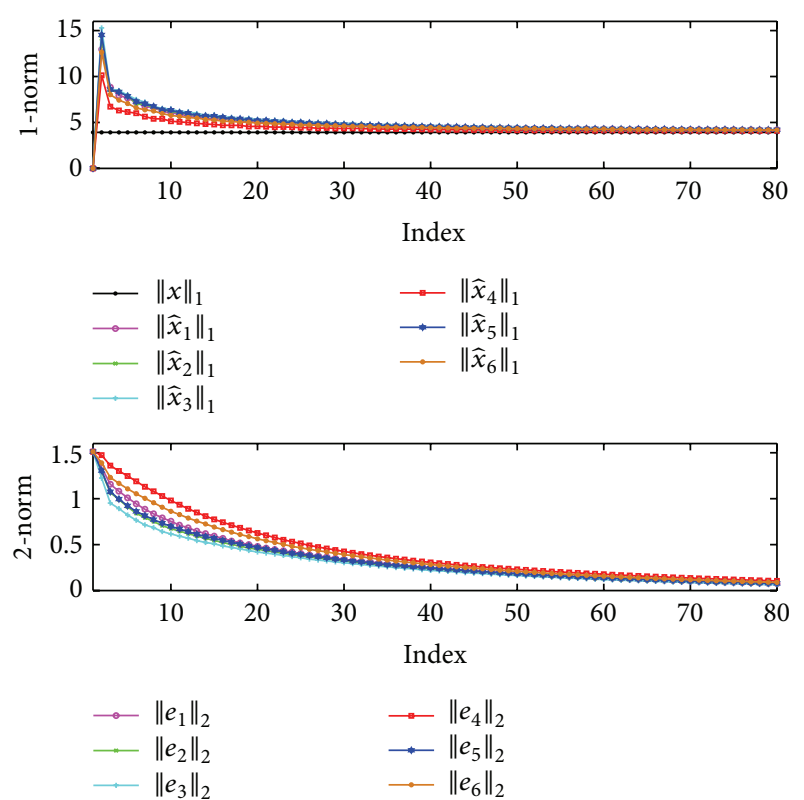

FIgURE 6: The evolution of $\left\|\widehat{x}_{i}\right\|_{1}(i=1,2, \ldots, 6)$ and $\left\|e_{i}\right\|_{2}(i=$ $1,2, \ldots, 6)$.

4.3. Sparse Signal Estimation Using KCIF-PM in All-to-All Network. In this part, an extreme example is presented to demonstrate the ability of Algorithm 1 to fuse the measurements in the whole network. Assume that each node only gets a one-dimensional measurement, that is, $H_{i} \in \mathbf{R}^{1 \times 256}$ with its entries sampled according to $\mathbf{N}(0,1 / 72)$. Every node
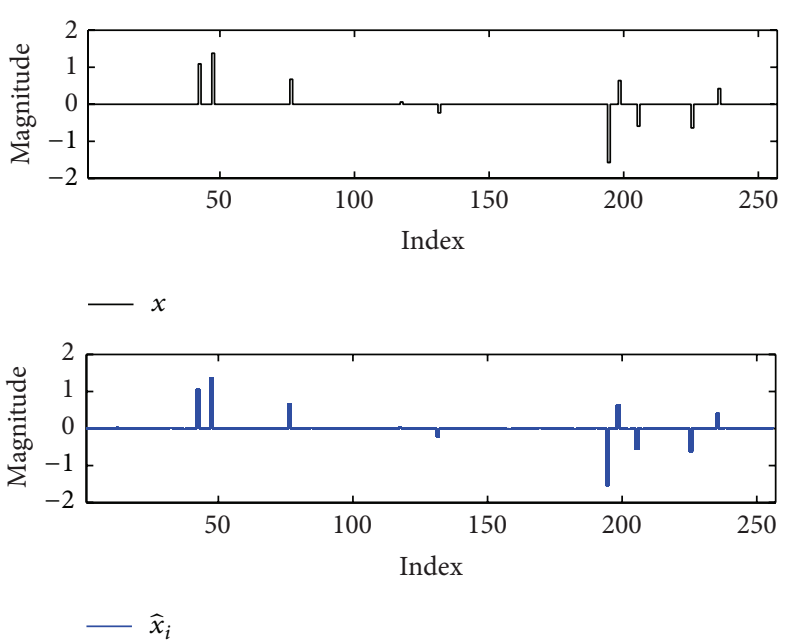

Figure 7: The sparse signal $x$ and its estimates $\widehat{x}_{i}$ using Algorithm 1 in all-to-all network.
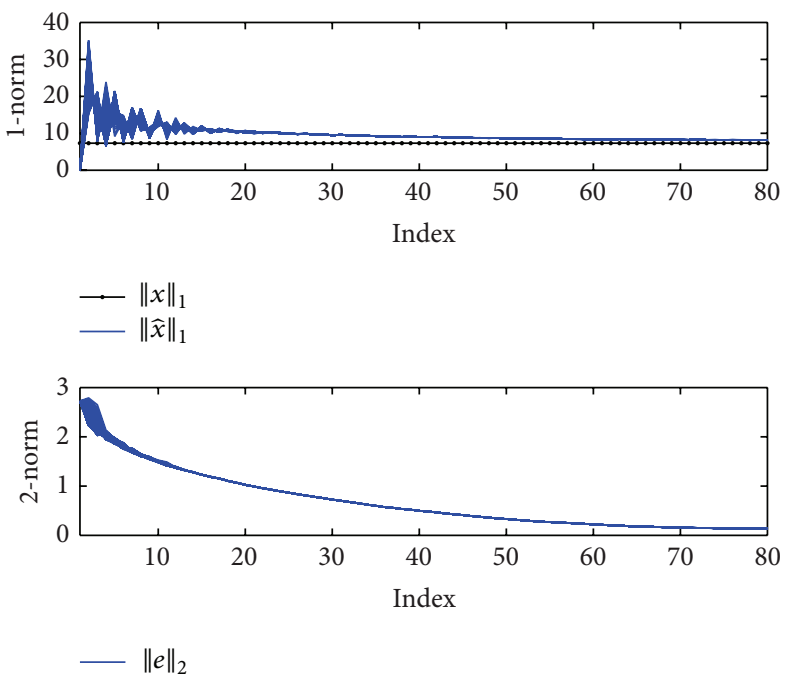

FIgUre 8: The evolution of $\left\|\widehat{x}_{i}\right\|_{1}$ and $\left\|e_{i}\right\|_{2}$ in all of nodes in the allto-all network.

communicates with all the others. Set parameters $\sigma_{i}^{2}=150+$ $7 k$ and $\epsilon=0.2$. As shown in Figures 7 and 8, the 1-norm of all of $\widehat{x}_{i}$ reaches to a consensus gradually and converges to $\|x\|_{1}$. $\left\|e_{i}\right\|_{2}$ converges to zero, which means all of the node filters are stable, and satisfactory estimates of the actual signal $x$ can be obtained.

\section{Conclusion}

This paper has demonstrated an effective approach to the problem of distributed sparse signal estimation in sensor networks. The filter has been designed by introducing the pseudomeasurement equation into the Kalman-consensus filter. A dynamic parameter selection strategy has been discussed to improve the performance of the existed algorithm. Some numerical examples have verified the effectiveness of the proposed method. In the future, we will try to test our 
KCIF-PM algorithm in some real-world applications, such as visual surveillance with multiple cameras or dynamic sparse signal tracking in wireless networks.

\section{Conflict of Interests}

The authors declare that there is no conflict of interests regarding the publication of this paper.

\section{Acknowledgments}

This work was supported by National Natural Science Foundation of China (Grant no. 61305128) and the Fundamental Research Funds for the Central Universities of China (Grant no. 3132014214 ).

\section{References}

[1] E. J. Candès, J. Romberg, and T. Tao, "Robust uncertainty principles: exact signal reconstruction from highly incomplete frequency information," IEEE Transactions on Information Theory, vol. 52, no. 2, pp. 489-509, 2006.

[2] T. T. Cai and L. Wang, "Orthogonal matching pursuit for sparse signal recovery with noise," IEEE Transactions on Information Theory, vol. 57, no. 7, pp. 4680-4688, 2011.

[3] G. Chinn, P. D. Olcott, and C. S. Levin, "Sparse signal recovery methods for multiplexing PET detector readout," IEEE Transactions on Medical Imaging, vol. 32, no. 5, pp. 932-942, 2013.

[4] E. Karseras, K. Leung, and W. Dai, "Tracking dynamic sparse signals using Hierarchical Bayesian Kalman filters," in Proceedings of the IEEE International Conference on Acoustics, Speech and Signal Processing (ICASSP '13), pp. 6546-6550, Vancouver, Canada, 2013.

[5] N. Vaswani, "Kalman filtered compressed sensing," in Proceedings of the IEEE International Conference on Image Processing (ICIP '08), pp. 893-896, San Diego, Calif, USA, October 2008.

[6] A. Carmi, P. Gurfil, and D. Kanevsky, "Methods for sparse signal recovery using Kalman filtering with embedded pseudomeasurement norms and quasi-norms," IEEE Transactions on Signal Processing, vol. 58, no. 4, pp. 2405-2409, 2010.

[7] A. Charles and C. Rozell, "Dynamic filtering of sparse signals using reweighted $\ell_{1}$," in Proceedings of the IEEE International Conference on Acoustics, Speech and Signal Processing (ICASSP '13), pp. 6451-6455, Vancouver, Canada, 2013.

[8] F. Cattivelli, C. Lopes, and A. Sayed, "Diffusion strategies for distributed Kalman filtering: formulation and performance analysis," in Proceedings of the Cognitive Information Processing, pp. 36-41, Santorini, Greece, 2008.

[9] F. S. Cattivelli and A. H. Sayed, "Diffusion strategies for distributed Kalman filtering and smoothing," IEEE Transactions on Automatic Control, vol. 55, no. 9, pp. 2069-2084, 2010.

[10] P. Di Lorenzo and A. H. Sayed, "Sparse distributed learning based on diffusion adaptation," IEEE Transactions on Signal Processing, vol. 61, no. 6, pp. 1419-1433, 2013.

[11] R. Olfati-Saber, "Distributed Kalman filtering for sensor networks," in Proceedings of the 46th IEEE Conference on Decision and Control (CDC '07), pp. 5492-5498, Los Angeles, Calif, USA, December 2007.

[12] R. Olfati-Saber, "Kalman-Consensus filter: optimality, stability, and performance," in Proceedings of the 48th IEEE Conference on Decision and Control held jointly with 28th Chinese Control Conference (CDC/CCC '09), pp. 7036-7042, Shanghai, China, December 2009.

[13] E. J. Candes and M. B. Wakin, "An introduction to compressive sampling: a sensing/sampling paradigm that goes against the common knowledge in data acquisition," IEEE Signal Processing Magazine, vol. 25, no. 2, pp. 21-30, 2008.

[14] D. Kanevsky, A. Carmi, L. Horesh, P. Gurfil, B. Ramabhadran, and T. N. Sainath, "Kalman filtering for compressed sensing," in Proceedings of the 13th Conference on Information Fusion (Fusion '10), pp. 1-8, Edinburgh, UK, July 2010. 


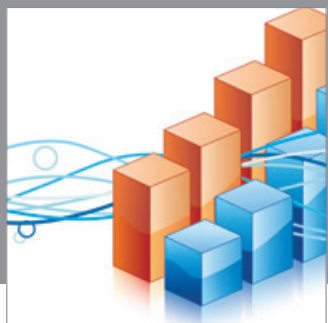

Advances in

Operations Research

mansans

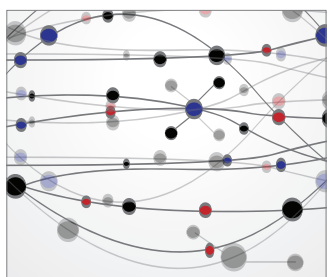

The Scientific World Journal
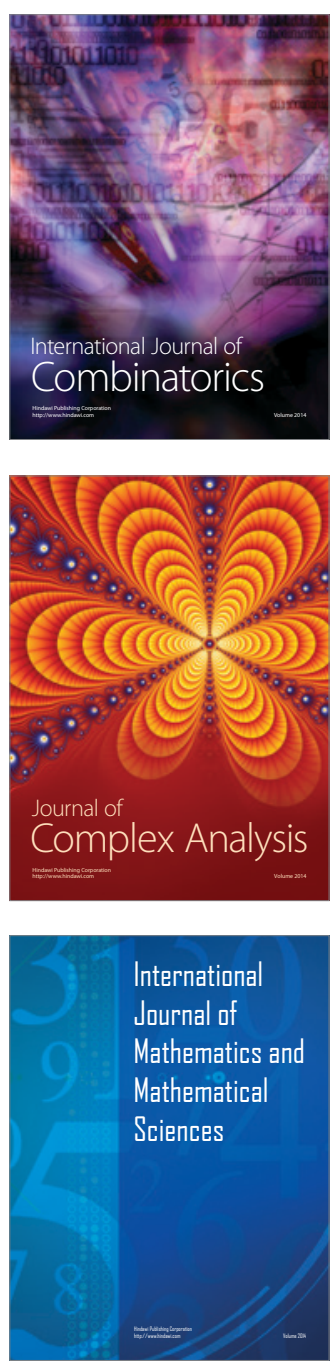
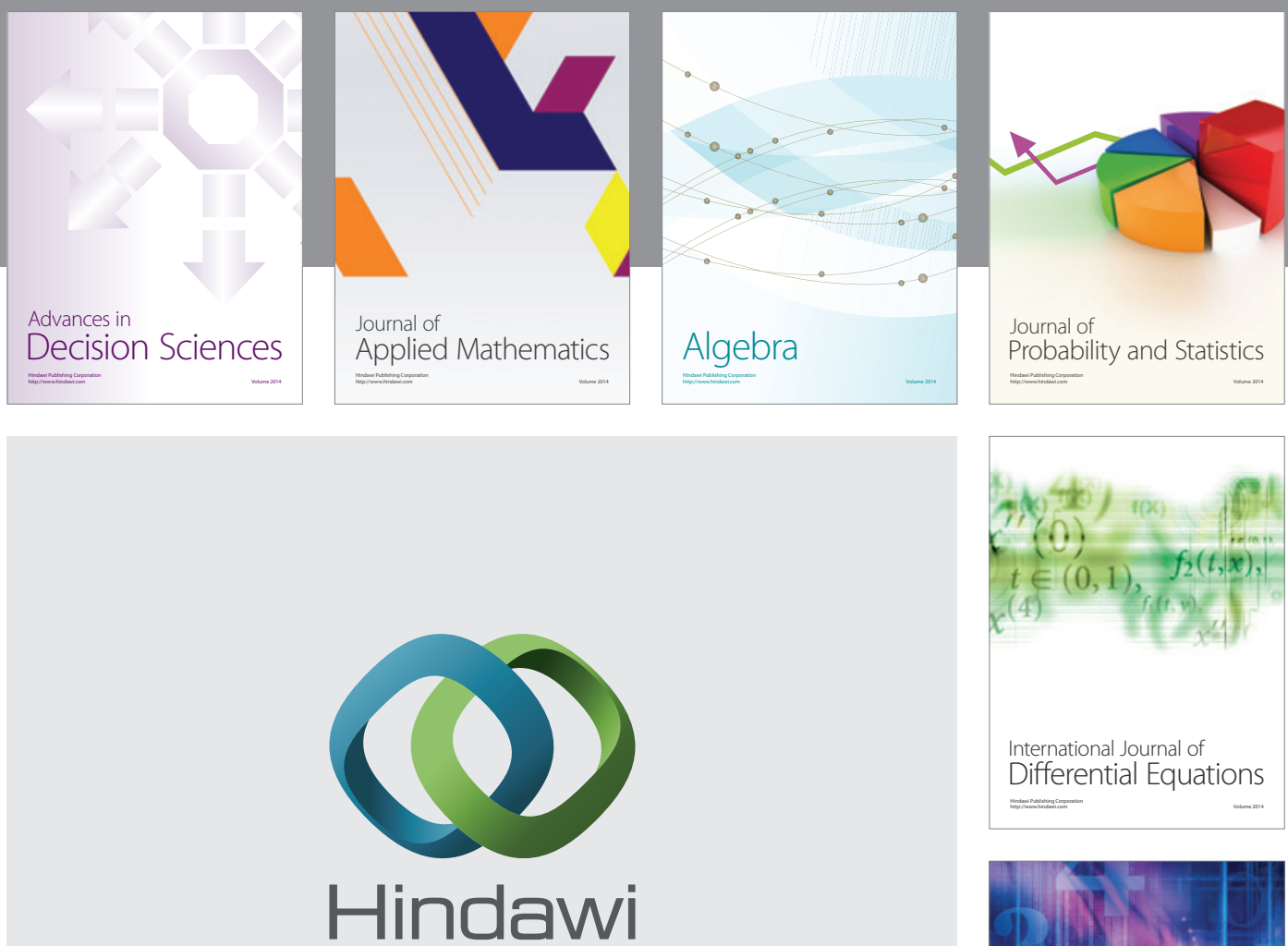

Submit your manuscripts at http://www.hindawi.com
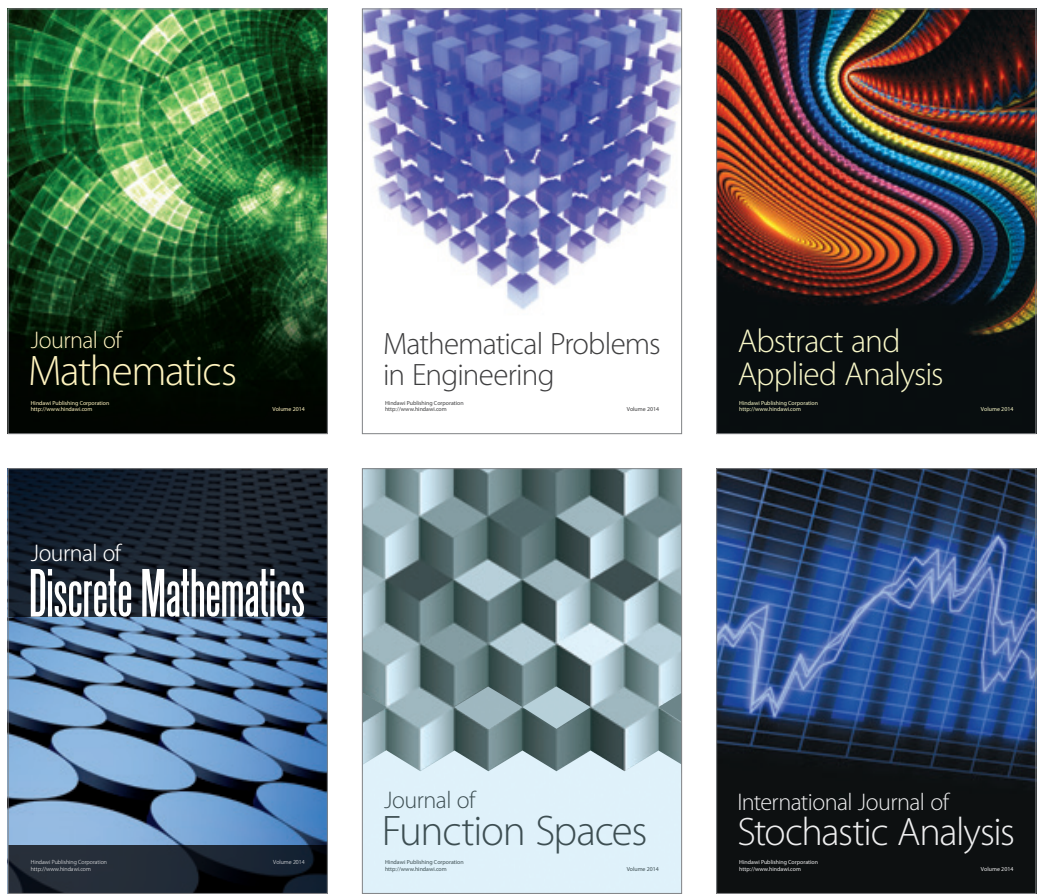

Journal of

Function Spaces

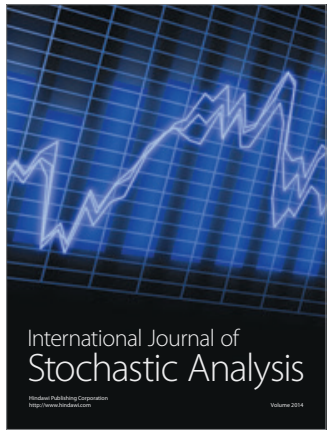

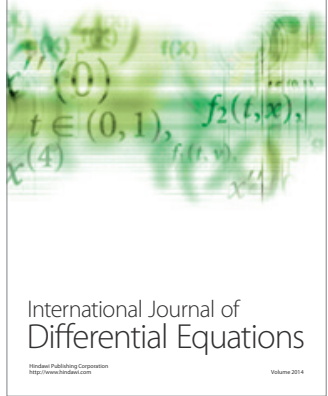
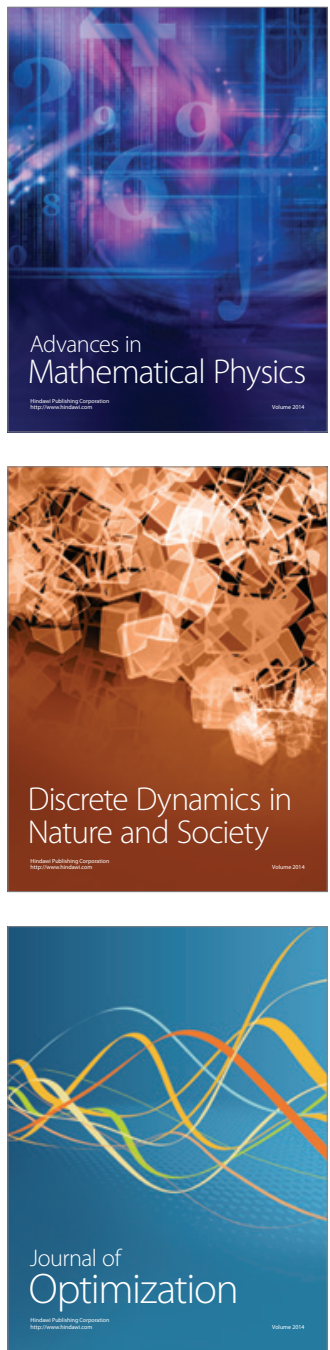